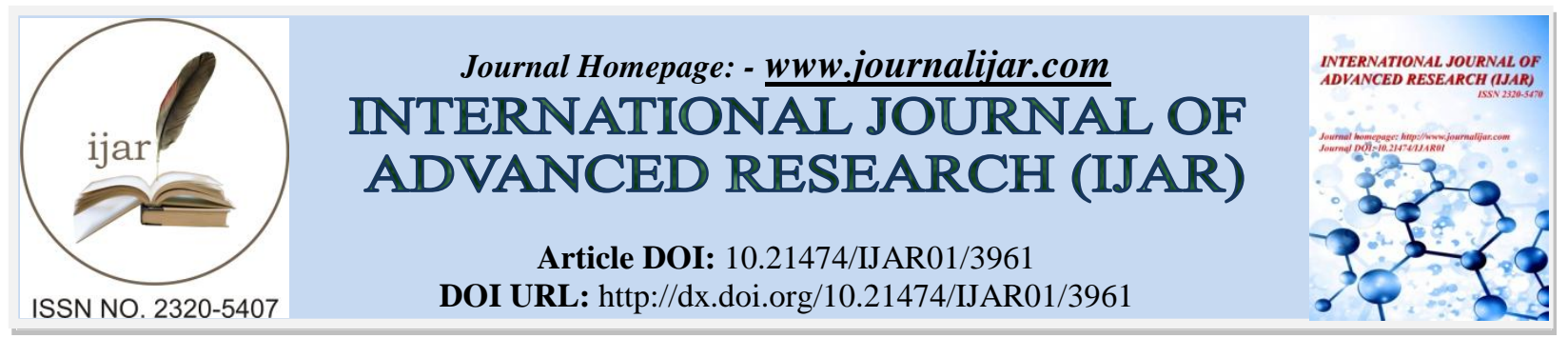

RESEARCH ARTICLE

\title{
AN INTRODUCTION TO INDICES IN ORTHODONTICS.
}

\section{"Dr. Pradeep Subbaiah ${ }^{1}$, Dr. Dhakshahini M. R ${ }^{2}$ and Dr. Anitha S.R ${ }^{3}$.}

1. lecturer department of orthodontics, JSS dental college \& hospital mysore-15.

2. vice principal, professor \& HOD department of prosthodontics JSS dental college \& hospital mysore-15.

3. pedodontic $\mathrm{s}$ and preventive dentistry.

\section{Manuscript Info}

Manuscript History

Received: 15 February 2017

Final Accepted: 19 March 2017

Published: April 2017

\section{Abstract}

Copy Right, IJAR, 2017,. All rights reserved.

Index According to A.L. Russell, an index is defined as 'A numerical value describing the relative status of the population on a graduated scale with definite upper and lower limits which is designed to permit and facilitate comparison with other population classified with the same criteria and method. ${ }^{1}$

An index is an expression of clinical observation in numerical values, which is used to describe the status of the individual or group with respect to a condition being measured. - E.M.Wilkins ${ }^{2}$.

In the orthodontic context index is described as - 'A rating or categorizing system that assigns a numeric score or alphanumeric label to a person's occlusion.'

Requirements of ideal orthodontic index are - (Jamison H.D. and Mc Millan R.S) ${ }^{3}$

Simple.

Reliable and reproducible.

Objective and yield quantitative data.

Differentiate between handicapping and non-handicapping malocclusions.

Measure degree of handicap.

Quick examination.

Amenable to modifications.

Usable either on patient or on study model.

Types Of Indices -According To Who ${ }^{4}$

\begin{tabular}{|l|l|l|}
\hline SI.no. & Indices & subclassification \\
\hline 1. & Occlusal Classification & Angle's classification by Angle in $1899^{5}$ \\
\cline { 3 - 3 } & & Incisor classification by Ballard and Wayman, $1964^{6}$ \\
\hline 2. & $\begin{array}{l}\text { Skeletal classification by Houston et } \\
\text { al, } 1993\end{array}$ & \\
\hline 3. & Malocclusion & Occlusal index by Summers $1966^{7}$ \\
\cline { 3 - 3 } & & $\begin{array}{l}\text { Handicapping Malocclusion Assessment Record (HMAR) } \\
\text { by Salzmann, 1968 }\end{array}$ \\
\hline 4. & Treatment assessment & Little's irregularity index by Little $1975^{8}$ \\
\hline
\end{tabular}

Corresponding Author:- Pradeep subbaiah.

Address:- lecturer department of orthodontics, JSS dental college \& hospital mysore-15. 


\begin{tabular}{|l|l|l|}
\hline & & Peer Assessment rating by Richmond et al, $1992^{9}$ \\
\hline \multirow{2}{*}{5.} & Cleft Outcome & GoslonYardstick by Mars et al, $1987^{10}$ \\
\cline { 3 - 3 } & & Year olds' Index by Atack et al, 1997 \\
\hline 6. & Periodontal & Plaque Index by Stilness \& Loe, 1964 \\
\cline { 3 - 3 } & & Gingival Index. by Loe \& Stilness, 1963 \\
\hline
\end{tabular}

Types of indices (w. C. Shaw ajo 1995) ${ }^{11}$

There are five types of index, each for a distinct purpose. Indeed it is the purpose rather than content or conventions of an index that distinguishes it.

\begin{tabular}{|c|c|c|}
\hline S.I NO & INDEX & EXAMPLES \\
\hline 1 & $\begin{array}{l}\text { Diagnostic classification- Angle's classification with its subclasses } \\
\text { used to describe incisor and buccal segment relationships separately } \\
\text { allows ease of communication between orthodontists. }\end{array}$ & $\begin{array}{l}\text { ANGLE'S CLASS I,CLASS II, } \\
\text { CLASS III }\end{array}$ \\
\hline 2 & $\begin{array}{l}\text { Epidemiologic indices- These indices record every trait in a } \\
\text { malocclusion to allow estimation of the prevalence of malocclusion in } \\
\text { a given population. Other indices of this type score tooth alignment in } \\
\text { a way that allows study of tooth irregularity and periodontal disease, } \\
\text { or treatment stability }\end{array}$ & $\begin{array}{l}\text { i) Epidemiologic registration of } \\
\text { malocclusion by Bjork, Krebs, } \\
\text { and Solow } \\
\text { ii)The FDI method } \\
\text { iii)Summer's occlusal index. }\end{array}$ \\
\hline 3 & $\begin{array}{l}\text { Treatment need (treatment priority) indices - Allow categorization of } \\
\text { malocclusion according to the level of treatment need. These indices } \\
\text { yield a score for each trait or component that is then weighted to } \\
\text { calculate an overall score. A simpler method of assigning treatment } \\
\text { priority is to establish a list of conditions or traits in categories that } \\
\text { denote the extent to which treatment is considered necessary. }\end{array}$ & $\begin{array}{l}\text { i) Draker's HLD index. } \\
\text { ii) Grainger's treatment priority } \\
\text { index. } \\
\text { iii) Salzman's handicapping } \\
\text { malocclusion assessment. } \\
\text { iv) IOTN. }\end{array}$ \\
\hline 4 & $\begin{array}{l}\text { Treatment outcome indices- Several indices have been developed to } \\
\text { evaluate treatment success. PAR index described later has been } \\
\text { developed specifically for this purpose. Summer's index has also been } \\
\text { used to assess the outcome of treatment. }\end{array}$ & \\
\hline 5 & $\begin{array}{l}\text { Treatment complexity index - At present no index has been described } \\
\text { to specifically measure treatment complexity but the desirability of } \\
\text { such an index in public health orthodontics is recognized and efforts } \\
\text { are presently underway to develop one. }\end{array}$ & \\
\hline
\end{tabular}

The methods of recording and measuring malocclusion can be broadly divided into two types- qualitative and quantitative -Tang and Wei AJO Apr $1993^{12,13}$

1. Qualitative Methods Of Recording Malocclusion:

Angle's method of classifying malocclusion in $1899^{5}$ [But Case pointed out that Angle's method disregarded the relationship of the teeth to the face and although malocclusion was a three-dimensional problem, Angle's system had only taken into account anteroposterior deviations in the sagittal plane. When Gravely and Johnson tested reliability, Angle's system was unreliable, including difficulty associated with asymmetry between left and right sides, or where tooth movements had occurred because of factors such as crowding and premature loss of deciduous teeth.]

Massler \& Franel's index ${ }^{16}$ recording the no. of displaced/rotated teeth in 1951.

Draker's HLD Index in 1960.

Occlusal feature index of Poulton \& Aaronson that records lower anterior crowding, cuspal inter-digitation, overjet and overbite.

Malocclusion severity index by Grainger in $1961^{17}$

Summer's Occlusal index in $1966^{7}$

Treatment Priority Index by Grainger in $1967^{18}$

Salzmann HMAR in $1968 .{ }^{19}$

2. Quantitative Methods Of Measuring Malocclusion:

In 1951 Massler and Frankel ${ }^{16}$ made the initial attempt to develop a quantitative method of assessing malocclusion. The total number of displaced or rotated teeth was the basis for the evaluation of prevalence and incidence of malocclusion in population groups. 
In 1959, VanKirk and Pennell proposed the malignment index, which involved the grading of tooth displacement and rotation quantitatively.

Draker developed the handicapping labiolingual deviation index (HLDI) in 1960

Poulton and Aaronson proposed the occlusal feature index in 1961. Since only four features of occlusion were measured this index was considered incomplete

\section{Evolution of Indices:-}

About the year 1800, people started to think that certain arrangements of the human teeth constituted perfection. This "idea" in the minds of men was that this configuration was normal and hence "ideal" occlusion. Soon, the notion developed that what was not ideal was not normal and was therefore abnormal!

In 1889, Edward H. Angle ${ }^{5}$ came on the scene and declared that the relationship of the first molars defined proper occlusion and stated that arrangements other than his definition were abnormal and by inference malocclusion.

In 1938, Henry Klein developed the DMFT (decayed, missing, filled) scale that was and is still the ultimate in simplicity in measuring dental conditions for large numbers of people.

In 1960, H. L. Draker ${ }^{14}$ suggested a different approach in which selected deviations from ideal were scored and weighted. He called it the Handicapping Labio-lingual Deviation index or HLD Index.

Grainger developed the malocclusion severity estimate (MSE) ${ }^{15}$, in the Burlington Research Center. It can be used either on models or on patients. Validity and reproducibility were high. However, there were at least three possible shortcomings of the MSE, namely: (1) the index was derived from data of 12-year-old patients and therefore might not be valid for earlier stages of dental development in the deciduous and mixed dentitions; (2) the MSE score didn't reflect all measurements that were accumulated and (3) the absence of any occlusal disorder was not scored as zero. The occlusal index (OI) was developed by Summers ${ }^{7}$ in 1966 and was based on the malocclusion severity estimate, with attempts to remedy its shortcomings.

The first shortcoming of the MSE could be remedied by scoring for each stage of dental development (i.e., deciduous, mixed, and permanent dentition stages) and different scoring forms were used for subjects in each stage. The second shortcoming was remedied by considering the scores of all syndromes in arriving at the final OI score. The MSE considered only the score of the syndrome with the highest score, but in the OI, the other scores were also considered by adding half of the sum of the remaining scores to the highest score among the seven syndromes.

The third shortcoming was remedied by adjusting for normality, so that the absence of any occlusal disorder would be scored as zero.

Grainger, in 1967, modified the MSE to develop the treatment priority index (TPI). Grainger described the index as a method of assessing the severity of the most common types of malocclusion, and hence, provided a means of ranking patients according to the severity of malocclusion, the degree of handicap, or their priority of treatment

In 1968, Salzmann developed the handicapping malocclusion assessment record (HMAR) ${ }^{19}$. The assessment forms and the definition of handicapping malocclusion presented were officially approved by the Council on Dental Health of the American Dental Association, and the Board of Directors of the American Association of Orthodontists Others who worked on this included Massler and Frankel, VanKirk and Pennell, Bjork et al, Summers, Freer and Adkin, Ingervall and Ronnerman, and Helm.

In 1972, Harvey Peck and Sheldon Peck developed a diagnostic index for assessing tooth shape deviations was for spatial analysis of existing or potential malocclusions.

In 1976, The Champus program, the Armed Forces of the United States contracted with the National Research Council to organize a work force to define "seriously handicapping orthodontic conditions" An excellent committee of highly qualified workers in the field labored for some time but was unable to produce such a definition.

The malocclusion index problem arises because of the need to identify which patient's treatments will be paid for with tax dollars. Both the civilian (Medicaid) and military (Champus) programs in the United States require that "need" be demonstrated. Need is defined as "medically necessary handicapping malocclusion" in Medicaid parlance. 
The "WHO/FDI Basic Method for Recording of Malocclusion"20 was published in 1979 to establish an assessment format to determine the prevalence of malocclusion and to estimate treatment needs of a population.

In 1985, the AAO formally recalled that action and specifically declared that they were opposed to the use of any index of malocclusion to identify patients needing treatment. Since that declaration, no American orthodontist has produced any work on these indexes.

In 1986, Cons, a Public Health dentist, is the only American dentist to produce any kind of an index since then. Cons approached the index problem from purely the appearance standpoint and developed the Dental Aesthetic Index. They generally used the opinions of the lay public as to what constituted unacceptable dental arrangements from the aesthetic standpoint. The Dental Aesthetic Index has been accepted by the World Health Organization as a screening tool.

The Medicaid statutes, as described in The State Medicaid Manual in April 1988, direct each state to provide orthodontic treatment for medically necessary handicapping malocclusion.

In 1989, California was sued for failing to comply with the orthodontic provisions of the Medicaid statutes. An orthodontist was offered a contract for $20 \mathrm{hrs}$ to draft a narrowly defined criteria and guidelines for determining medically necessary orthodontic treatment. He used the HLD Index but intended to add the 3 missed-out features viz., deep impinging bites traumatizing the soft tissue of the palate, crossbites of individual anterior teeth destroying the soft tissue and a large maxillary protrusion with reasonably aligned teeth - buck tooth which is an extreme social handicap. It also included ectopic eruption suggested by Draker. Hence the HLD (CalMod) Index came into being.

In 1994, California was sued again and led to some needed changes in the regulations. Overjet greater than $9 \mathrm{~mm}$ and reverse overjet greater than $3.5 \mathrm{~mm}$ were inserted as a qualifying exception.

Grade Index Scale for assessment of treatment need: The Swedish National Board developed a four grade index scale designed to determine whether a patient's malocclusion, to classify patients ranging from those who need very urgent orthodontic treatment to those of little need to identify who fall within the scope of treatment in the Swedish public dental services. However, the criteria were not well defined and the cut off points were vague.

Espeland produced a new approach in Norway for their mixture of public and private funding of treatment to determine the level of public health copayment that the patient may be entitled to, i.e., total reimbursement for severe malocclusion with cleft lip and palate, and partial or nil reimbursement for malocclusions considered minor.

In 1987, the British Orthodontic Standards Working Party convened a series of meetings with a group of 10 experienced Orthodontists to develop an index of treatment outcome from study casts of various treated and untreated occlusions. Richmond designed a ruler to allow analysis of a set of study casts in 2 minutes (approx). The result was the Peer Assessment Rating or PAR index.

More recently in 1989, Brook and Shaw (EJO) developed the index of orthodontic treatment need (IOTN), which consisted of the dental health component and the esthetic component. In the dental health component, occlusal traits thought to contribute to the longevity and satisfactory functioning of the dentition were defined and placed in five grades, with clear cut-off points. The esthetic component consisted of a 10-point scale illustrated by a series of numbered photographs that were rated for attractiveness. The index was found to be satisfactorily valid and reproducible.

In 1992, Haeger also developed a static occlusal analysis in centric occlusion based on ideal interarch and intraarch relationships and called it ideal tooth relationship index.

Malocclusion Severity Estimate - Grainger (1960-61) ${ }^{17}$

Measurements: 1) Overjet 2) Over bite 3) Anterior open bite 4) Congenitally missing maxillary incisors 5) First molar rotation 6) Posterior cross bite 7)Tooth displacement - actual and potential.

Six malocclusion syndromes defined:

i. Positive overjet, anterior open bite

ii. Positive overjet, posterior over bite, distal molar relationship and posterior cross bite with maxillary teeth buccal to mesio distal teeth.

iii. Negative overjet, mesial molar relationship, posterior cross bite. 
iv. Tooth displacement.

v. Posterior open bite.

vi. Mesial molar, over jet, over bite, cross bite, midline diastema, midline deviation.

vii. Mesial molar, mixed dentition analysis and actual tooth displacement.

A Peck and Peck index for assessing tooth shape deviations (1972 Apr AJO) ${ }^{21}$

Odontometry is the science of measuring the size and proportion of teeth.

Harvey Peck and Sheldon Peck devised an index using which lower incisor crowding can be assessed from tooth size measurements obtained either from the mouth or from plaster casts. This spatial analysis of existing or potential malocclusions is an important diagnostic tool. Naturally well-aligned mandibular incisors possess are significantly smaller mesiodistally and larger faciolingually.

Index = Mesiodistal width of mandibular incisor / Faciolingual width of mandibular incisor $\mathrm{x} 100$

$\mathrm{MD} / \mathrm{FL}$ index is a numerical expression of the crown shape as viewed incisally.

$\mathrm{MD} / \mathrm{FL}$ index of well-aligned mandibular central incisors $=88.4 \pm 4.3$ lateral incisors $=90.4 \pm 4.8$.

Clinical guidelines for the maximum limit of desirable MD/FL index values for the lower incisors:

- Mandibular central incisor 88-92

- Mandibular lateral incisor 90-95

\section{Applications:-}

1. Prediction of unerupted tooth size similar to mixeddentition analyses by Moyer's and Nance

2. Assessment of tooth size - arch size compatibility within the same arch.

3. Assessment of tooth size compatibility between the two arches - Bolton's ratio.

This index has been well applied in studies of approximal and occlusal tooth wear. Reproximation ("stripping") is described as a clinical procedure for correcting tooth shape deviations.

WHO or FDI CLASSIFICATION - $1979^{20}$ :

1. Gross anomalies

2. Dentition - missing, supernumerary, malformed incisor, ectopic eruption

3. Space - Diastema, crowding, spacing

4. Occlusion -

a) Incisal - maxillary mandibular overjet, cross bite, deep bite, open bite, midline shift.

b) Lateral segment - AP relation, open bite, posterior cross bite.Subjective judgement of orthodontic treatment - Not necessary, doubtful, necessary, urge

Index of Orthodontic Treatment Need (IOTN) - W. C. Shaw AJO $1995^{22}$

It ranks malocclusion in terms of the significance of various occlusal traits for the person's dental health and perceived esthetic impairment, with the intention of identifying those persons who would be benefited the most from orthodontic treatment.

\section{The Dental Health Component of IOTN:-}

Each occlusal trait thought to contribute to the longevity and the satisfactory functioning of the dentition is defined and placed into five grades, with clear cut-off points between the grades. The index recognizes the most severe anomaly or dental diseases specific to that site. Summing scores for a series of individual traits is not performed. Thus, multiple minor variations, each of which is unimportant with respect to dental health, cannot be added together to place a person in a higher grade.

\section{The Esthetic Component of IOTN:-}

The esthetic component consists of a 10-point scale, illustrated by a series of numbered photographs from an earlier study, which were rated for attractiveness by laypersons and selected as being equidistantly spaced through the range of scores. A rating is allocated for overall dental attractiveness rather than specific morphologic similarity to the photographs. The value arrived at gives an indication of the patient's treatment need on the grounds of esthetic impairment, and by inference reflects the sociopsychologic need for orthodontic treatment. When applied in the clinical setting, the patient's lips are retracted with self-retaining lip retractors, and a rating allocated. The time taken to record both the dental health and esthetic components by an experienced examiner is approximately 1 minute. 
However, if several minor anomalies require examination to identify the most severe, grade allocation may take up to 3 minutes.

Resource allocation and planning for limited public and other third-party resources for orthodontic care, to direct resources to cases most likely to derive benefit from treatment.

1. Monitoring and promoting standards.

2. Modification of the PAR Index there is also a place for an index of treatment complexity since this could be used for triage or allocation where groups of providers of varying levels of competence coexist.

3. Patient identification and referral. Access to orthodontic care is often determined by the general dentist or pedodontist.

Informed consent - The patient should also be provided with a candid description of the risks and stability of treatment, and thus be in a position to make a more balanced decision.

Disadvantage:

3. Risk of insensitivity and misjudgment of the needs of the individual patient. None of the available systems can solve the dilemma of patients with minor irregularities about which they are deeply concerned and which may therefore have a bearing on self-esteem and self-confidence.

4. Peer Assessment Rating or PAR index Richmond $1987^{23}$ :

5. A scoring system was developed and a ruler designed to allow analysis of a set of study casts in approximately 2 minutes.

6. Individual scores for the components of alignment and occlusion are finally summed to calculate an overall score. Thus, a score of zero would indicate perfect alignment and occlusion and higher scores (rarely beyond 50) indicate increasing levels of irregularity.

7. The index is applied to both the start and the end of treatment study casts, and the change in the total score reflects the success of treatment in achieving overall alignment and occlusion.

8. The PAR index and IOTN were applied to the pretreatment and posttreatment casts. The mean percentage reduction in PAR score was $52 \%$ and $21 \%$ of cases were unimproved or worse as a result of orthodontic treatment. When outcome was related to choice of appliance, combined upper and lower fixed appliances produced the best standard of treatment, mean reduction in PAR being $71 \%$. Cases with a borderline need for treatment (IOTN grade 3) were more liable to have unsuccessful treatment. A lack of improvement or worsening of the malocclusion occurred in $34 \%$ of cases. the results obtained by spring type removable appliances were significantly inferior to those gained by fixed appliances.

9. Senior orthodontists with greater experience gained better results than trainees, and some centers provided a better overall standard of care than others, seemingly reflecting the differing aspirations of the heads of different centers

10. The investigation of treatment standards is a more challenging step for the specialty.

11. Poor standards of orthodontic care when nonspecialists provide treatment

12. Risk benefit balance was shown to be extremely uncertain for malocclusion with borderline need for treatment pointing to the high level of competence that would be required if moderate malocclusion is to be treated at all.

13. Indices of treatment outcome, however, can also be a powerful educational tool for the individual provider. For a provider's treatment standards to be acceptable, the percentage reduction in PAR score for a sample of cases should exceed a level agreed by consensus. the results obtained by spring type removable appliances were significantly inferior to those gained by fixed appliances.

\section{The measurements made are:-}

1. Overjet

2. Overbite

3. Midline relation

4. Buccal Occlusion

5. Upper anterior crowding

Ideal tooth relationship index - Haeger AJO May 1992 It is a static occlusal analysis in centric occlusion based on ideal interarch and intraarch relationships for quantitating the degree of interdigitation of the teeth. 


\section{Applications:-}

1. evaluating the results of orthodontic treatment

2. posttreatment stability, settling, relapse

3. different orthodontic treatment modalities and

4) assess treatment effects of orthodontic appliances

\section{Characteristic Features:-}

* Index scores were generated for the entire dentition, which could be divided into anterior, posterior, interarch, and intraarch relationships.

* Index scores were determined at various time intervals so the longitudinal changes could be studied.

* The number of potential ideal relationships varied depending on the number of teeth included, i.e., extraction cases and inclusion of second molars.

* No difference in scores if only first molars were included.

* The relationships were scored only when they were correct, and no range of "normal" was incorporated.

* If a buccal segment interdigitated mesially or distally to the Class I position, contacts were still counted as being present since functional inclined plane relationships were of primary interest.

* Models with congenitally missing teeth, questionable articulation, malformed teeth or broken or chipped teeth were not included in this study.

* Third molars were not included because of variability in form and occurrence.

* Deciduous teeth were excluded.

\section{Results:-}

Evaluation of treated malocclusions

\section{Total ITRI score:-}

An initial of $26 \%$

1. Increased to $52 \%$ at the end of orthodontic treatment and

2. Continued to improve to $59 \%$ during the retention and post retention periods.

3. Anterior segment scores were higher (64\%) than posterior segment scores $(44 \%)$.

4. The intraarch relationships of the anterior segment registered the highest $(75 \%)$, whereas the lingual cusp relationships of the posterior segment had the lowest scores (35\%).

* Buccal relationships in the posterior segment produced higher scores than the lingual segment.

* Lingual cusp relations showed the smallest improvement as a result of orthodontic treatment but the largest amount of retention and post retention settling.

* Before treatment there were differences between the index scores of the various malocclusion groups.

* After orthodontic treatment, no difference in ITRI scores existed between the four malocclusion groups. This was true for both segments and all components.

* During the retention and postretention periods, the posterior intraarch scores of the Class II, Division 1 malocclusions increased an additional 15 percentage points.

The system allowed the computation of a total index score that could be divided into arch segments and components of these segments so that details of occlusal changes during treatment and posttreatment could be followed.

Analysis of treatment results showed that anterior segments improved more than posterior segments, and buccal relations are handled better than lingual relations. It appears orthodontists do a better job correcting discrepancies that are more highly visible.

Occlusal relationships after orthodontic treatment were improved to approximately the same degree regardless of the type of malocclusion and, thereafter, showed similar settling and relapse.

Profitt And Ackermann - $1973^{24}$

1. Alignment - Ideal, crowding, spacing, mutilated

2. Profile - Mesiodistal prominence, mesiodistal recession, lip profile relative to nose and chin. (Convex, straight, concave)

3. Posterior cross bite 
4. Saggital plane - Angle's classification

5. Bite depth - Anterior or posterior open or deep bite

Evaluation Of Malocclusion Indices:-

* The reproducibility or reliability of an index is the ability to produce the same score or measurement when one or more examiners measure the same case at the same or at a different time.

* The validity of an index can be defined as its ability to accurately measure what it purports to measure.

* Bias, or systemic error of an index or measurement is the magnitude and direction of its tendency to measure something other than what was intended. The score of an unbiased index should accurately reflect the intended characteristics. For malocclusion indices, severity of malocclusion and the priority for treatment based on need would be the intended characteristics. An index could be precise but biased. In such a case, the score will be reproducible but not an accurate portrayal of the occlusion.

* Weights were first introduced to put different emphasis on various measurements.

* Only the OI had developed different scoring schemes and scoring forms for patients in different stages of dental development, i.e, deciduous dentition, mixed dentition, and permanent dentition. Later, weighting systems were also included in the OI, the TPI, and the HMAR.

Hermanson and Grewe tested the precision and bias of five malocclusion indices including the HMAR, the OI, the TPI, and two other indices. Their results showed that only the OI and the TPI demonstrated nonsignificant interexaminer variability at the $1 \%$ level, and that the most precise and unbiased index would be the OI or the TPI.

Grewe and Hagan ${ }^{25}$ compared the HMAR, the OI, and the TPI for precision and bias when used in the same population. The results showed that all three indices were highly reproducible. When bias or systematic error was evaluated, the results indicated that the OI described the clinical standard most accurately. Therefore, of the three indices tested in the study, no one index can be selected over the others with regard to precision, but the OI would be the index of choice, with regard to having the least amount of bias.

Summers tested the validity of three indices ${ }^{26}$ : The CHAMPUS index, the HMAR, and the OI. The OI was found to be the most valid among the three indices. When validity during time was tested, decreased scores were noted in the CHAMPUS index and the HMAR but not in the OI.

In conclusion, The Summers occlusal index appear to have the least amount of bias has the highest validity during time and is best correlated with clinical standards.

\section{Conclusion:-}

A good method of recording or measuring malocclusion is important for documentation of the prevalence and severity of malocclusion in population groups. This kind of data is not only important for the epidemiologist, but also for those who plan for the provision of orthodontic treatment in a community or for the training of orthodontic specialists. If the method is universally accepted and applied, data collected from different population groups can be compared.McLain and Proffit "occlusal problems cannot be defined solely in physical terms." They say that the psychosocial consequences due to unacceptable dental esthetics may be as serious, or even more serious, than the biologic problems

\section{Reference:-}

1. An assessment tool, named after A. L. Russell, a contemporary American dentist, that estimates the degree of periodontal disease ,January 2010 | ISBN: 9780199533015 ; 4735 pages.

2. Gen Index: Births: Rachel E M Wilkins

3. An index of malocclusion for use in multiphasic screening and epidemiological investigations.Jamison HC, McMillan RS., Ala J Med Sci. 1966 Apr;3(2):154-8.

4. World Health Organization. An international methodology for epidemiological study of oral disease. Manual No.5: Epidemiological studies of periodontal disease. First draft. Geneva, 1966.

5. Angle EH. Classification of malocclusion. Dent Cosmos 1899; 41:248-64.

6. Ballard CF, Wayman JB. A report on a survey of the orthodontic requirements of 310 army apprentices. Dent Pract Dent Rec 1965; 15: 221-6.

7. Summers CJ, Arbor A. The Occlusal Index: A system for scoring and identifying occlusal disorders. Am J Orthod. 1971; 59(6):552-67.

8. Little RM. The Irregularity Index. Am J Orthod 1975; 68:554-63. 
9. Richmond S, Shaw WC, Roberts CT, Andrews M. The PAR Index (Peer Assessment Rating): Methods to determine orthodontic treatment in terms of improvement and standards. Eur J Orthod 1992; 14(3):180-87.

10. Mars M, Plint DA, Houston WJB, Bergland O, Semb G. The Goslon Yardstick: A new system of assessing dental arch relationship in children with unilateral cleft lip and palate. Cleft Palate J 1987; 24:4:314-322.

11. The use of occlusal indices: a European perspective. Shaw WC, Richmond S, O'Brien KD.Am J Orthod Dentofacial Orthop. 1995 Jan;107(1):1-10.

12. Tang ELK, Wei SHY. Recording and measuring malocclusion: A review of the literature. Am J Orthod Dentofacial Orthop 1993; 103: 344-51.

13. Agarwal A, Mathur R. An Overview of Orthodontic Indices.World J Dent 2012; 3(1):77-86.

14. Draker HL. Handicapping labio-lingual deviations: A proposed index for public health purposes. Am J Orthod 1960; 46:295-305.

15. Grainger RM. Malocclusion Severity estimate Progress Report, Series VI. Burlington Orthodontic Research Centre. 1960-61:9-1

16. Massler M, Frankel JM. Prevalence of malocclusion in children aged 14-18 years. Am J Orthod 1951; 37:75168.

17. Grainger RM. Malocclusion Severity estimate Progress Report, Series VI. Burlington Orthodontic Research Centre. 1960-61:9-11.

18. Grainger RM. Orthodontic Treatment Priority Index. 1967. Public Health Service Publication No. 1000, Series 2, No. 25, US Government Printing Office, Washington DC.

19. Salzmann JA. Handicapping malocclusion assessment to establish treatment priority. Am J Orthod. 1968; 54(10):749-69.

20. 15. Baume, LJ; Horowitz, HS; Summers, CJ; Dirks, BO; Brown, WAB; Carlos, JP. A method for measuring occlusal traits. Int Dent J 1973; 23:530-37.

21. An index for assessing tooth shape deviations as applied to the mandibular incisors, Harvey Peck, Sheldon Peck, April 1972Volume 61, Issue 4, Pages 384-401

22. Brook PH. Shaw WC. The development of an index of orthodontic treatment priority. Eur J Orthod 1989; 20:309-20.

23. Richmond S, Shaw WC, Roberts CT, Andrews M. The PAR Index (Peer Assessment Rating): Methods to determine orthodontic treatment in terms of improvement and standards. Eur J Orthod 1992; 14(3):180-87.

24. Ackerman, J.L., Proffit, W.R. (1969). The characteristics of malocclusion. A modern approach to classification and diagnosis. Am J Orthod Dentofac Orthop. 56: 443-454

25. Grewe, J.M., Hagan, D.V. (1972). Malocclusion indices. A comparative evaluation, Am J of Orthod. 61: 286294.

26. Summers, C.J. (1972). Test for validity for indices of occlusion. Am J Orthod. 62 (4): 428-429. 